\section{$\mathbf{m} / \mathbf{S}$}

médecine/sciences $1990 ; 6: 624-5$

\title{
Le muscle : système modèle en biologie du développement
}

\section{François Gros}

\author{
RÉFÉRENCES
}

1. Lemonnier M, Libri D, Mouly V, Fiszman M. Les tropomyosines. médecine/sciences $1990 ; 6: 645-52$

2. Buckingham ME. The control of muscle gene expression. A review of molecular studies on the production and processing of primary transcripts. Brit Med Cell 1989 ; $45: 608$.

3. Maire P, Gautron S, Hakim V, Gregori C, Mennecier F, Kahn A. Characterization of three optional promoters in the 5' region of the human aldolase A gene. $J \mathrm{Mol} B i o l$ $1987 ; 197$ : 425.

4. Libri D, Fiszman MY. Épissage différentiel des transcrits musculaires. médecine/sciences $1990 ; 6$ : 626-34.

5. Gros F, Montarras D, Pinset C, Mouly

$\mathrm{V}$. Hétérogénéité myoblastique et filiation myogénique. médecine/sciences $1990 ; 6$ : 245-51.

6. Ott M-O, Robert B, Buckingham M. Le muscle, d'où vient-il ? médecine/sciences 1990 ; 6 : 653-63.

7. Alonso S. Des facteurs de régulation spécifiques de la myogenèse. médecine/sciences $1990 ; 6: 635-44$.

8. Davis RL, Cheng P-F, Lassar AB, Weintraub $H$. The MyoD DNA binding domain contains a recognition code for muscle-specific gene activation. Cell 1990 ; $60: 733$.

9. Kingston RE. Transcription control and differentiation: the HLH family c-myc and C/EBP. Current opinion in Cell Biology $1989 ; 1: 1081$.

10. Benezra R, Davis RL, Lockshon D, Turner DL, Weintraub $\mathrm{H}$. The protein Id : a negative regulator of helix-loop-helix DNA binding proteins. Cell 1990 ; 61 : 49.

11. Montarras D, Pinset C, Chelly J, Kahn A, Gros F. Expression of $M y o D 1$ coincides with terminal differentiation in determined but inducible muscle cells. EMBO J 1989 ; $8: 2203$.

\section{ADRESSE}

F. Gros : professeur à l'Institut Pasteur et au Collège de France. Unité de biochimie, Institut Pasteur, 25, rue du Docteur-Roux, 75015 Paris, France.

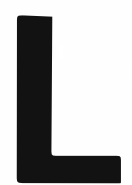

ongtemps le muscle commun des vertébrés, qui constitue, avec leur ossature, la masse pondérale prédominante, ne fut considéré par les biologistes à tout prendre que comme une machine à produire du mouvement. Le mouvement n'est-il pas pour Aristote, avec la reproduction, l'une des caractéristiques essentielles de la vie? L'analogie "muscle-moteur " ou " ossature-levier " contribuera beaucoup à l'essor des fameuses théories cartésiennes sur l'animal-machine. Avec l'étude anatomo-physiologique des muscles au XIXe siècle, la découverte des premières maladies "musculaires" par Duchenne, puis les premiers travaux sur les synapses neuromusculaires et la plaque motrice vont se préciser et s'affiner nos connaissances relatives à la commande biologique du mouvement. L'ère biochimique dans l'étude de ce tissu familier s'ouvrira dans le sillage des recherches du laboratoire de St. Gyorgii sur la myosine et sur l'actine, de Bailey sur les tropomyosines [1] et d'Ebashi sur les troponines, etc. Peu à peu, l'appareil contractile va se trouver ainsi "décortiqué " et l'on sait comment les histologistes, puis l'école des biophysiciens anglais, notamment grâce à Andrew Huxley, parviendront à expliquer, dans un schéma unitaire, le mécanisme moléculaire de la contraction.

Pour autant (et cette situation a prévalu jusque dans le début des années 70) le modèle musculaire est demeuré quelque peu en retrait des grandes démarches qui, après la dernière guerre, ont caractérisé l'essor de la génétique du développement. Ainsi, chez les organismes supérieurs, l'archétype cellulaire dans les travaux sur la différenciation a très longtemps été l'érythrocyte (à la suite sans doute des idées de L. Pauling sur les "maladies moléculaires").

La situation a considérablement changé lorsque, il y a une quinzaine d'années divers biologistes ont démontré que la cellule embryonnaire précurseur du tissu musculaire, ou myoblaste, pouvait se différencier en une ministructure plurinucléée contractile in vitro, le myotube, puis en myofibre en l'absence de toute innervation. Le programme de développement musculaire pouvait désormais se prêter à une analyse biochimique indépendamment du nerf, tout en offrant des perspectives fort intéressantes à l'étude des modulations épigénétiques associées à l'innervation.

Les premières lignées myoblastiques à développement continu ont ensuite été isolées, rendant désormais possible l'étude des mécanismes de régulation génétique qui accompagnent leur transformation en myofibres contractiles. Enfin, cette étude n'allait pas tarder à bénéficier des techniques de l'ADN recombinant de sorte que, dans le début des années 80 , les biologistes moléculaires étaient en mesure d'appréhender certains des mécanismes clés du développement (myogenèse) de ce tissu. Compte tenu de la très grande variété des protéines constituant l'appareil contractile (sarcomère), l'étude du déterminisme génétique de la myogenèse se ramenait dans la plupart des cas à analyser les éléments régulateurs qui commandent à l'activation ou à la mise au repos des gènes de ces protéines "sarcomériques".

Une première constatation allait dès lors s'imposer : l'étonnante diversité des mécanismes régulateurs ainsi mis en œuvre (pour une revue récente voir [2]) ; la fabrique d'une unité sarcomérique à un instant précis du développement 
repose en effet sur des stratégies aussi différentes et contrastées que : la coexpression d'isogènes (actines), l'activation "en cascade " des gènes d'une même famille formant des groupes de liaison sur le chromosome (grandes sous-unités des myosines), l'activation différentielle d'un gène à promoteurs multiples (aldolases) (voir par exemple [3]) ou encore l'épissage alternatif (chaînes légères de myosines, tropomyosines...), etc. Pourquoi tant d'isoformes sont-elles synthétisées lors du développement ? Quelle est la logique sous-jacente à cette diversité dans les mécanismes régulateurs au cours de l'ontogenèse, alors que toutes ces protéines concourent à une même fonction (contraction) et se retrouvent finalement assemblées en un même organelle, le sarcomère ? Toujours est-il que ceux qui s'intéressent aux relations entre phylogenèse et ontogenèse trouveront là un modèle de choix. Avec deux articles sur le déterminisme génétique dans la formation des tropomyosines au cours du développement aviaire [Marguerite Lemonnier [1] ; Domenico Libri et Marc Fiszman [4]], ce numéro illustre bien l' "exquise" complexité des mécanismes ontogéniques, mais aussi la formidable précision avec laquelle s'opère, dans la myogenèse programmée, le passage d'une isoforme à une autre.

On verra que le muscle des vertébrés ne mobilise pas moins de quatre gènes de structure pour fabriquer ses $\alpha$ - et $\beta$-tropomyosines, mais que parmi les très nombreux transcrits ainsi formés, certains subissent des épissages différentiels conduisant à des isoformes multiples peut-être adaptées à telle ou telle étape de l'ontogenèse ou à telle fonction physiologique.

Mais, tandis que l'on commence à percevoir de quelle manière une cellule ontogéniquement déterminée, le myoblaste, "mobilise" son clavier génétique pour se différencier, l'étude de la myogenèse prend un " second souffle ": les biologistes viennent probablement de mettre en effet le doigt, pour la première fois, sur les éléments régulateurs intervenant dans les stades les plus précoces de la formation du tissu: ceux que les embryologistes désignent sous le nom $\mathrm{m} / \mathrm{s} n^{\circ} 7$ vol. 6 , septembre 90 de "détermination". On sait en effet, grâce à leurs travaux, que le muscle de squelette dérive d'un territoire particulier de l'embryon, le mésoderme. Il est connu que les cellules de mésoderme s'organisent en groupements réguliers à distribution antéropostérieure : les somites, lesquels subissent des remaniements précédant la formation d'autres territoires cellulaires qui engendrent finalement l'ossature, les cellules du derme et le muscle. Les myoblastes somitiques convergent en vagues successives vers les muscles du tronc ou des membres [5]. Une revue exhaustive de ce numéro [6] fait le point de l'état actuel des connaissances relatives à l'induction du mésoderme, phénomène résultant d'une interaction entre les deux premiers feuillets existants de l'embryon avant gastrulation, l'ectoderme et l'endoderme. De remarquables progrès ont été accomplis dans la caractérisation des facteurs d'induction diffusibles s'apparentant au $\beta$-TGF et au FGF et l'on commence à entrevoir les premières réponses moléculaires à l'induction mésodermique.

Mais la question " cardinale ", certes, est bien de savoir comment, au niveau des rouages génétiques, les cellules mésodermiques deviennent des myoblastes. Quels événements génétiques dictent, à côté des influences épigénétiques, un tel " choix "? Quatre gènes régulateurs $(M y o D$, Myogénine, Myf5, Myf6) plus un cinquième moins bien identifié (Myd) sont impliqués dans œ choix [7]. L'ADN complémentaire de chacun d'eux, séparément, suffit à convertir, par transfection, des fibroblastes multipotents en myoblastes. Tous les éléments régulateurs que codent ces gènes sont apparentés quant à leur organisation (domaine acide, domaine basique servant à reconnaître les enhancers spécifiques du phénotype musculaire, et surtout existence d'une conformation identique où deux $\alpha$-hélices sont séparées par une boucle courte (conformation HLH) [8].

Le plus remarquable, en cette affaire, est que ces protéines régulatrices s'associent en hétérodimères à la fois entre elles et avec d'autres éléments porteurs du motif HLH dont l'exis- tence est ubiquitaire : les protéines E12 ou E47 [8, 9]. Un dimère MyoD-E12 stimulera tel enhancer tandis qu'une combinatoire distincte, impliquant E12 ou E47 avec une autre protéine à motif $\mathrm{HLH}$, agira vraisemblablement sur une autre, etc. Poussant plus avant leur quête, les biologistes ont même réussi à identifier des éléments particuliers, les "facteurs ID" [10] qui, en s'associant à E12 ou E47, les empêchent de coactiver les divers éléments régulateurs de la famille "MyoD ". Bien des inconnues subsistent : la première est : dans quel ordre (selon quelle séquence temporelle) interviennent ces gènes ? Une question sur laquelle les travaux de Montarras et al. [11] à l'aide de lignées de $\mathrm{Mb}$ inductibles jettent déjà un éclairage très intéressant, mais qui est abordée aussi avec une extrême précision par M. Buckingham et ses collaborateurs en analysant les transcrits des gènes de la "famille MyoD " dans des somites de souris en développement, grâce aux techniques de l'hybridation in situ [6]. Il existe, à cet égard une assez bonne convergence entre les données obtenues in vitro et in vivo. Elles s'accordent pour dire que le gène $M y f 5$ entrerait assez tôt en fonction, sans doute avant $M y o D$ et $M y o^{-}$ génine, Myf6 ayant au contraire un rôle plus tardif. Mais il faudra bien expliquer quel est en tout cela le facteur déclencheur qui met en branle ces gènes de détermination et comment ces derniers interagissent entre eux.

On le voit, l'étude de la différenciation musculaire est un thème en plein essor. Son étude n'a pas fini de nous étonner : l'emploi des animaux transgéniques et de la recombinaison homologue nous promet encore bien des découvertes. Mais surtout, les données qu'elle fournit devraient permettre d'aborder sous un angle désormais différent bien des aspects de la pathologie du muscle. Celle-ci, depuis l'identification des gènes directement concernés par la myopathie de Duchenne ou par l'amyotrophie spinale, progresse également très vite, situation illustrant de façon exemplaire à quoi peut conduire une interpénétration raisonnée de la biologie moléculaire et de la médecine 